\title{
Effect of dietary cholesterol with or without saturated fat on plasma lipoprotein cholesterol levels in the laboratory opossum (Monodelphis domestica) model for diet-induced hyperlipidaemia
}

\author{
Rampratap S. Kushwaha ${ }^{1}$, Jane F. VandeBerg ${ }^{2}$ and John L. VandeBerg ${ }^{2}$ \\ ${ }^{1}$ Department of Physiology and Medicine, Southwest Foundation for Biomedical Research, San Antonio, Texas 78245-0459, \\ USA \\ ${ }^{2}$ Department of Genetics, Southwest Foundation for Biomedical Research, San Antonio, Texas 78245-0459, USA \\ (Received 25 November 2003 - Revised 3 March 2004 - Accepted 9 March 2004)
}

\begin{abstract}
Laboratory opossums (Monodelphis domestica) show extreme genetic variability in their responsiveness to dietary lipids; a great proportion of the genetic variability in responsiveness is due to a single major gene. To determine whether the major gene for dietary response detected by genetic analysis in opossums is responsive to dietary cholesterol or dietary saturated fat, or a combination of both, we used males and females of susceptible and resistant lines of laboratory opossums that were 5 to 7 months old. The animals were challenged with three different experimental diets (high-cholesterol diets with or without high saturated fat from lard or coconut oil) and plasma lipoproteins were measured. Plasma and VLDL+LDL-cholesterol concentrations increased several-fold when the animals were fed the diet containing elevated cholesterol $(P<0.001)$ or elevated cholesterol and fat $(P<0.001)$ and differed between the two lines when they were fed high-cholesterol diets with or without fat $(P<0.001)$. Plasma HDL-cholesterol concentrations were higher $(P<0.05)$ in animals of the resistant line than in the susceptible line when they were fed the basal diet (550 (SEM 30) v. 440 (SEM 20) mg/l) and when they were fed the low-cholesterol and high-fat diet (600 (SEM 30) v. 490 (SEM 30) mg/l). Dietary coconut oil and lard had similar effects on plasma lipoprotein cholesterol concentrations in the susceptible line of opossums. A reduction in dietary cholesterol by $50 \%$ with either the lard or coconut oil blunted the plasma cholesterol response. The results from the present studies suggest that the major gene for dietary response previously detected by genetic analysis in laboratory opossums affects the response to dietary cholesterol but not to saturated fat.
\end{abstract}

Cholesterol: Lipoproteins: Susceptible and resistant lines of opossums: High-density lipoproteins: Very low-density lipoproteins + low-density lipoproteins

Laboratory opossums (Monodelphis domestica) show extreme variability in their plasma cholesterol levels when challenged with a high-cholesterol and high-fat (HCHF) diet (Rainwater \& VandeBerg, 1992). The plasma cholesterol variability is due to VLDL+LDLcholesterol only. Low-responding animals show a slight increase in VLDL+LDL-cholesterol, whereas highresponding animals show up to a 100 -fold increase in VLDL+LDL (mainly in LDL) cholesterol. Lipoprotein phenotype data from a large number of animals fed two diets were previously analysed by complex segregation analysis (Rainwater et al. 2001). The results suggested that VLDL+LDL-cholesterol concentrations in animals fed the basal and the fat- and cholesterol-enriched diets are affected by two major genes (i.e. single genes with a large effect). The major gene detected for VLDL+LDLcholesterol concentration whilst feeding the basal diet explains $24 \%$ of the variability in VLDL+LDL-cholesterol concentration but does not influence VLDL+LDLcholesterol concentration when feeding the HCHF diet. The major gene locus for VLDL+LDL-cholesterol when feeding the $\mathrm{HCHF}$ diet explains $80 \%$ of the variability in VLDL + LDL-cholesterol concentrations in animals fed the HCHF diet. Since a single major gene is responsible for most of the genetic variability in VLDL+LDL-cholesterol response, the laboratory opossum is an excellent model for obtaining a genetic marker for responsiveness to diet. This genetic marker may be applicable to man or it may be useful in designing drugs to manage human diet-induced hyperlipidaemia. However, it is not known whether the differences in plasma lipoprotein cholesterol responses to diet between susceptible and resistant lines of opossums are due to the dietary fat or dietary cholesterol or a combination of both. It is generally believed that the

\footnotetext{
Abbreviations: ACAT, acyl-coenzyme A:cholesterol acyltransferase; HCHF, high-cholesterol and high-fat; SFBR, Southwest Foundation for Biomedical Research.

* Corresponding author: Dr Rampratap S. Kushwaha, fax +1 210670 3323, email kush@sfbr.org
} 
genes that regulate lipoprotein metabolism in response to dietary fat (saturated fatty acids) may be different from those that regulate lipoprotein metabolism in response to dietary cholesterol. Since plasma cholesterol concentration whilst being fed the HCHF diet is inherited as a major gene, we tested the hypothesis that the major gene is responsive to dietary cholesterol, to dietary fat, or to a combination of both. Therefore, the aim of the present study was to identify the lipid component of the diet to which the major gene responds in controlling VLDL+ LDL-cholesterol level in laboratory opossums. We also compared the effect of the type of saturated fatty acids (from lard and coconut oil) on plasma lipoprotein cholesterol response in animals from a susceptible line of opossums.

\section{Methods}

\section{Animals}

For these studies, we used partially inbred strains of the grey short-tailed opossum, a marsupial native to Brazil and adjoining countries. At the Southwest Foundation for Biomedical Research (SFBR), a breeding programme has led to the development of eleven partially inbred strains of laboratory opossums with inbreeding coefficients $>0.6$. Among these inbred strains, one high- and three low-responding strains were developed specifically for their responsiveness to dietary lipids. Two of these lines have been designated as ATHH (the high-responding or susceptible line) and ATHE (the low-responding or resistant line), respectively. For both experiments, we used eighty opossums from the susceptible line (ATHH stock) and forty opossums from the resistant line (ATHE stock). The selected animals were 5-7 months old. Initially, the animals were challenged with a HCHF diet, which contains $188 \mathrm{~g}$ fat $/ \mathrm{kg}(140 \mathrm{~g} \mathrm{lard} / \mathrm{kg})$ and $7 \cdot 1 \mathrm{~g}$ cholesterol $/ \mathrm{kg}$. After 6 weeks of consuming the HCHF diet, $1.0 \mathrm{ml}$ blood was collected from each animal. Fasting plasma was used for the measurement of total plasma cholesterol and triacylglycerols, and lipoprotein cholesterol concentrations. On the basis of cholesterol response data, the animals were assigned to experiments.

\section{Experimental diets}

The basal diet for the laboratory opossums was a commercial pelleted fox food (Reproduction Diet, Nutritionally
Complete Fox Food Pellets; Milk Specialties Co., New Holstein, WI, USA). This diet contained $100 \mathrm{~g}$ fat $/ \mathrm{kg}$ (Table 1; basal diet). The cholesterol content of the diet was $1.6 \mathrm{~g} / \mathrm{kg}$. Special diets were prepared from the fox basal diet by adding 7.5 litres tap water to $22.7 \mathrm{~kg}$ of pellets, together with fat and/or crystalline cholesterol (USB Corp., Cleveland, OH, USA). The ingredients were mixed in a Hobart food mixer (Hobart Corporation, Troy, $\mathrm{OH}$, USA), until all of the liquids were absorbed. Then the blend was passed through a meat grinder (Torrey open bowl grinder; Rodriguez Butcher Supplier, San Antonio, TX, USA) to produce soft pellets, and frozen at $-20^{\circ} \mathrm{C}$ to prevent spoilage. Table 1 summarises the fat and sterol contents of the diets.

\section{Experimental design}

Long-term experiment. We selected twenty-four animals from the susceptible line and twenty-six from the resistant line. This selection process ensured the availability of at least twenty animals that responded typically for their respective lines, and that survived this first stage of the experiment. However, during the course of the experiment, some animals died and there were at least ten animals in each group fed the last diet. Therefore, data for ten animals in each group are presented. However, the data for differences in the response of males and females are presented using all animals.

The selected laboratory opossums were returned to the basal diet and maintained on this diet for 10 weeks. By this time, plasma cholesterol levels had reached baseline levels, and the animals were started on the HCHF diet. This diet contained $188 \mathrm{~g}$ fat $/ \mathrm{kg}(140 \mathrm{~g}$ lard $/ \mathrm{kg})$ and $7 \cdot 1 \mathrm{~g}$ cholesterol $/ \mathrm{kg}$. After 6 weeks of consuming the HCHF diet, the animals were bled, and plasma and lipoprotein cholesterol concentrations were measured. Afterwards, animals were fed the basal diet for 10 weeks, after which time the plasma cholesterol levels had again reached baseline levels. Then the animals were challenged with a lowcholesterol and high-fat diet. This diet contained $188 \mathrm{~g}$ fat $/ \mathrm{kg}(140 \mathrm{~g} \mathrm{lard} / \mathrm{kg})$ and $1.4 \mathrm{~g}$ cholesterol $/ \mathrm{kg}$. After 6 weeks of consuming the low-cholesterol and high-fat diet, the animals were bled, and plasma and lipoprotein cholesterol concentrations were measured. Animals were then fed the basal diet for 10 weeks, after which the animals were fed a high-cholesterol and low-fat diet. This diet contained $108 \mathrm{~g}$ fat $/ \mathrm{kg}$ and $7 \cdot 1 \mathrm{~g}$ cholesterol $/ \mathrm{kg}$. After

Table 1. Fat and sterol content of opossum (Monodelphis domestica) diets ( $\mathrm{g} / \mathrm{kg}$; dry weight basis)

\begin{tabular}{|c|c|c|c|c|}
\hline Ingredient & Basal diet* & $\begin{array}{l}\text { High-cholesterol and } \\
\text { high-fat diet } †\end{array}$ & $\begin{array}{l}\text { Low-cholesterol and } \\
\text { high-fat diet } †\end{array}$ & $\begin{array}{c}\text { High-cholesterol and } \\
\text { low-fat diet* }\end{array}$ \\
\hline Basal fatł (dairy products) & 30 & 27 & 27 & 30 \\
\hline Maize oil & 23 & 21 & 21 & 32 \\
\hline Cholesterol & $1 \cdot 6$ & $7 \cdot 1$ & 1.4 & $7 \cdot 1$ \\
\hline Plant sterols & 0.5 & 0.4 & 0.4 & 0.5 \\
\hline
\end{tabular}

* Low-fat diets provided approximately $20 \%$ of the total energy from fat.

$\dagger$ High-fat diets provided approximately $40 \%$ of the total energy from fat.

$\ddagger$ Other ingredients of the basal diet included $(\mathrm{g} / \mathrm{kg})$ : protein, 310; crude fibre, 45; simple carbohydrates, 530 ; vitamins and minerals, $<20$.

$\S$ For coconut-oil diets, lard was replaced with coconut oil. 
6 weeks of dietary challenge with the high-cholesterol and low-fat diet, the animals were bled, and plasma and lipoprotein cholesterol and triacylglycerol levels were measured. The dietary treatment has been summarised in Table 2.

Short-term experiment. We selected forty animals from the susceptible line and divided them into four groups with ten animals in each group. These animals were challenged for 4 weeks with four diets to determine the effect of reduced level of cholesterol with lard or coconut oil. We also compared the effect of type of saturated fat from coconut oil and lard on the plasma cholesterol response in this experiment. The first diet was low-cholesterol and highlard $(3.55 \mathrm{~g}$ cholesterol $/ \mathrm{kg}, 140 \mathrm{~g}$ lard $/ \mathrm{kg})$. The second diet was low-cholesterol and high-coconut-oil (3.55 g cholesterol $/ \mathrm{kg}, 140 \mathrm{~g}$ coconut oil $/ \mathrm{kg}$ ). The third diet was high-cholesterol and high-lard (7.1 g cholesterol/kg, $140 \mathrm{~g}$ coconut oil $/ \mathrm{kg}$ ). The fourth diet was high-cholesterol and high-lard, the same as the HCHF diet (7.1 g cholesterol/ $\mathrm{kg}, 140 \mathrm{~g} \mathrm{lard} / \mathrm{kg}$ ). The dietary treatment of animals has been summarised in Table 2 .

The protocol of these experiments was approved by the Institutional Animal Care and Use Committee of the SFBR. The SFBR is accredited by the Association for the Assessment and Accreditation of Laboratory Animal Care and is registered with the US Department of Agriculture.

\section{Blood sampling}

Animals were fasted overnight and blood $(1.0 \mathrm{ml})$ was collected by cardiac puncture from animals anaesthetised with halothane (Halocarbon Laboratory, River Edge, NJ, USA), and placed in a $2 \mathrm{ml}$ EDTA-vacutainer tube (Becton Dickinson Labware, Franklin Lakes, NJ, USA). Experienced technical staff collecting blood routinely are able to obtain $0.8-1.0 \mathrm{ml}$ blood with a death rate of $<1 \%$ (Robinson \& VandeBerg, 1994; VandeBerg, 1999). Cardiac puncture can be conducted repeatedly over time on an animal without ill effects (Manis et al. 1992). Plasma was obtained by centrifugation.

\section{Plasma and lipoprotein cholesterol analysis}

Total plasma cholesterol was measured enzymically on a Ciba-Corning Express Analyser (Infolab, Round Rock, TX, USA) with reagents supplied by Boehringer Mannheim Diagnostics (Indianapolis, IN, USA). Samples having cholesterol levels that exceeded the highest calibrator (Polestar Laboratory, Escondido, CA, USA) were diluted with saline and were repeated. The assay for HDL-cholesterol was performed on the diluted sample also. The within-run $\mathrm{CV}$ was $2.0 \%$ and the between-run $\mathrm{CV}$ was $3.0 \%$. HDL-cholesterol was determined by precipitation of Apo B-containing lipoproteins (VLDL+LDL) by heparin- (Elkins-Sinn, Inc., Cherry Hill, NJ, USA) manganese chloride (Sigma Chemical Co., St Louis, MO, USA) according to a procedure specified by the Lipid Research Clinics Program (1974). The VLDL+LDLcholesterol concentration was calculated as the difference between the total plasma cholesterol and HDL-cholesterol concentration.

\section{Measurement of triacylglycerols}

Plasma triacylglycerols were measured enzymically on a Ciba-Corning Express Analyser (Infolab) with reagents supplied by Stanbio Laboratory (San Antonio, TX, USA).

\section{Data analysis}

Values in tables and figures are expressed as means and standard errors of the mean. The values for highand low-responding opossums were compared using ANOVA with repeated measures. Significance was set at $P<0.05$.

\section{Results}

Effect of dietary cholesterol and/or fat on plasma cholesterol

Plasma cholesterol concentrations of susceptible and resistant lines of laboratory opossums in response to dietary

Table 2. Dietary treatment of laboratory opossums (Monodelphis domestica) in long- and short-term experiments*

\begin{tabular}{lcc}
\hline & & Number of animals completing \\
the diet
\end{tabular}

* For details of diets and procedures, see Table 1 and p. 64.

†The animals died due to procedures for repeated bleedings. The body weight and feed consumption did not differ between the animals of the two lines. 
cholesterol and/or fat are shown in Fig. 1 (A). Plasma cholesterol concentrations increased significantly in highresponding animals fed diets containing elevated levels of cholesterol alone $(P<0.001)$, or cholesterol and fat $(P<0.001)$, but not fat alone. The increase in plasma cholesterol in animals of the susceptible line when fed the HCHF diet was more than 10-fold (8290 (SEM 920) $\mathrm{mg} / \mathrm{l})$ compared with when they were fed the basal diet (640 (SEM 40) $\mathrm{mg} / \mathrm{l}$ ). However, the increase in plasma cholesterol when the susceptible animals were fed the high-cholesterol and low-fat diet (3980 (SEM 930) mg/l) was 6-fold. Similarly, plasma cholesterol concentration increased significantly in resistant opossums when they
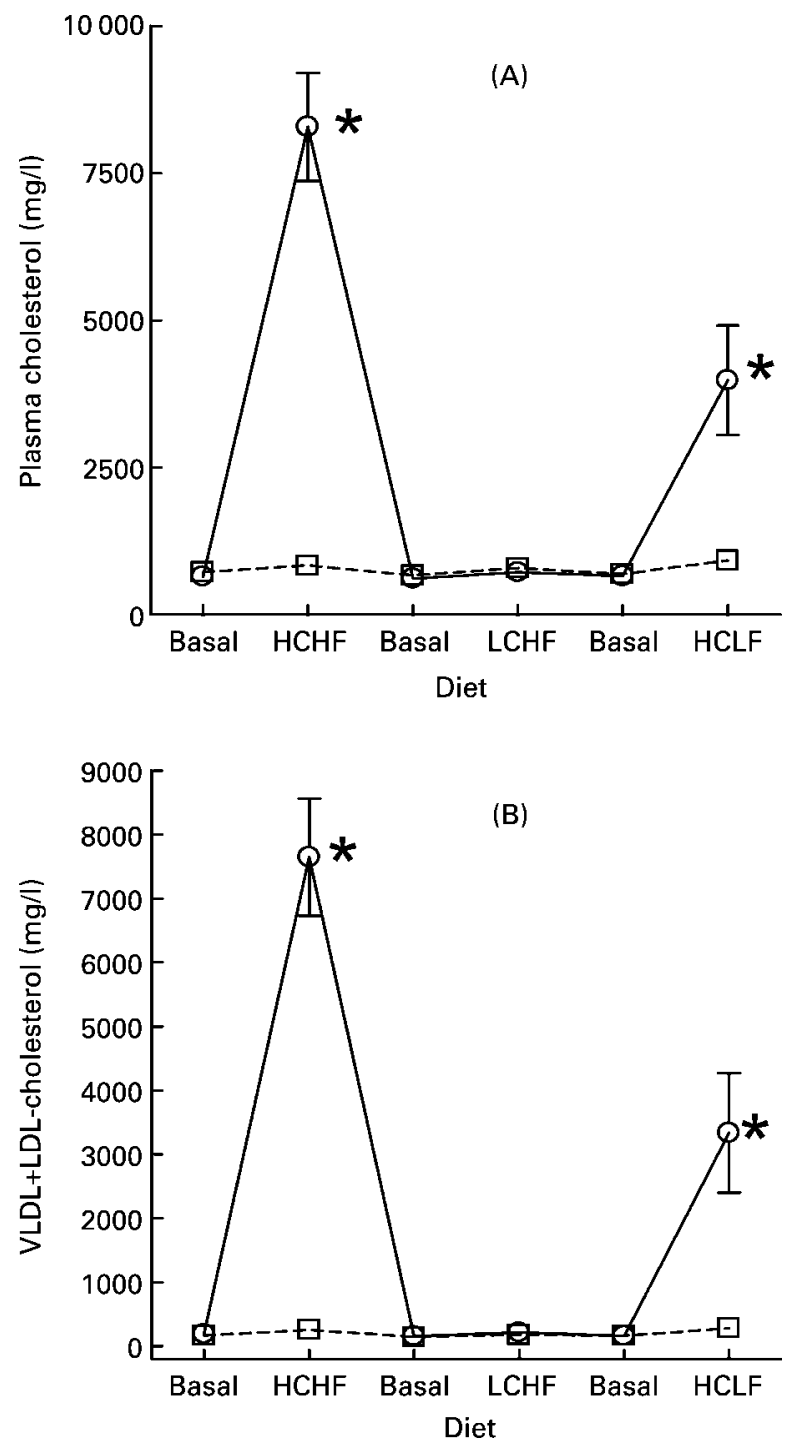

Fig. 1. Plasma cholesterol (A) and VLDL + LDL-cholesterol (B) concentrations of susceptible (-O-) and resistant $(-\square-)$ lines of laboratory opossums (Monodelphis domestica; ten in each group) in response to diets containing elevated cholesterol and/or fat. A basal diet was fed between each experimental diet. The experimental diets were: high-cholesterol and high-fat (HCHF); low-cholesterol and high-fat (LCHF); high-cholesterol and low-fat (HCLF) (see Table 1). Mean values are shown, with standard errors of the mean represented by vertical bars. * Mean values for susceptible and resistant lines were significantly different $(P<0.01)$. were fed diets containing elevated cholesterol levels $(P<0.05)$. However, the extent of increase was much less, ranging from 1.2-fold (720 (SEM 40) $\mathrm{mg} / \mathrm{l}$ when fed the basal diet $v .830$ (SEM 50) $\mathrm{mg} / \mathrm{l}$ when fed the HCHF diet) in response to elevated dietary cholesterol and fat to 1.4-fold (920 (SEM 60) mg/l) in response to elevated dietary cholesterol alone. Plasma cholesterol concentration did not differ between animals of the susceptible and resistant lines when they were fed the basal diet or the diet containing a high level of fat alone.

\section{Effect of dietary cholesterol and/or fat on plasma very low-density lipoprotein + low-density lipoprotein- cholesterol}

The VLDL+LDL-cholesterol-concentrations of laboratory opossums in response to diets containing high amounts of cholesterol and/or fat are shown in Fig. 1 (B). Plasma cholesterol in animals of the susceptible line increased significantly when they were fed the HCHF diet $(P<0.001)$, and the highcholesterol diet $(P<0.001)$, but not when they were fed the high-fat and low-cholesterol diet $(P=0 \cdot 15)$. VLDL+LDLcholesterol concentrations of the susceptible line fed the basal, HCHF, high-cholesterol and low-fat, and low-cholesterol and high-fat diets were 200 (SEM 20), 7650 (SEM 910), 3340 (SEM 930) and 220 (SEM 20) mg/l, respectively. The increase in plasma VLDL+LDL in susceptible animals when fed the HCHF diet was approximately 38-fold, whereas the increase in plasma VLDL+LDL-cholesterol when fed the high-cholesterol diet was approximately 17fold. In response to the HCHF diet, and the high-cholesterol and low-fat diet, more than $85 \%$ of the total plasma cholesterol existed in the form of VLDL+ LDL in high-responding opossums. Similarly, plasma VLDL+LDL-cholesterol increased significantly in the low-responding opossum line when fed the HCHF diet $(P<0.001)$, and the high-cholesterol and low-fat diet $(P<0.001)$, but not when fed the low-cholesterol and high-fat diet. VLDL+LDL-cholesterol concentrations of the resistant line when fed the basal, $\mathrm{HCHF}$, high-cholesterol and low-fat, and low-cholesterol and high-fat diets were 170 (SEM 10), 260 (SEM 30), 280 (SEM 40) and 180 (SEM 20) mg/l, respectively. The increase in plasma VLDL+LDL-cholesterol in the resistant line was much less by comparison with the susceptible line (1.5- to $1 \cdot 8$-fold). As a result, there was very little increase in the proportion of VLDL+LDL-cholesterol in the plasma. Plasma VLDL+LDL-cholesterol concentrations differed significantly between animals of the susceptible and resistant lines when they were fed the HCHF diet $(P<0.001)$ and the high-cholesterol and low-fat diet $(P<0.001)$.

\section{Effect of dietary cholesterol and/or fat on plasma high-density lipoprotein-cholesterol}

As shown in Fig. 2, resistant animals have significantly higher $(P<0.05)$ HDL-cholesterol (530 (SEM 19) mg/l) than susceptible animals (463 (SEM 13) $\mathrm{mg} / \mathrm{l}$ ) when fed the basal diet. There was an increase in plasma HDLcholesterol in both the susceptible and resistant lines for all three experimental diets $(P<0.05)$. The increases in plasma HDL-cholesterol concentrations in susceptible 


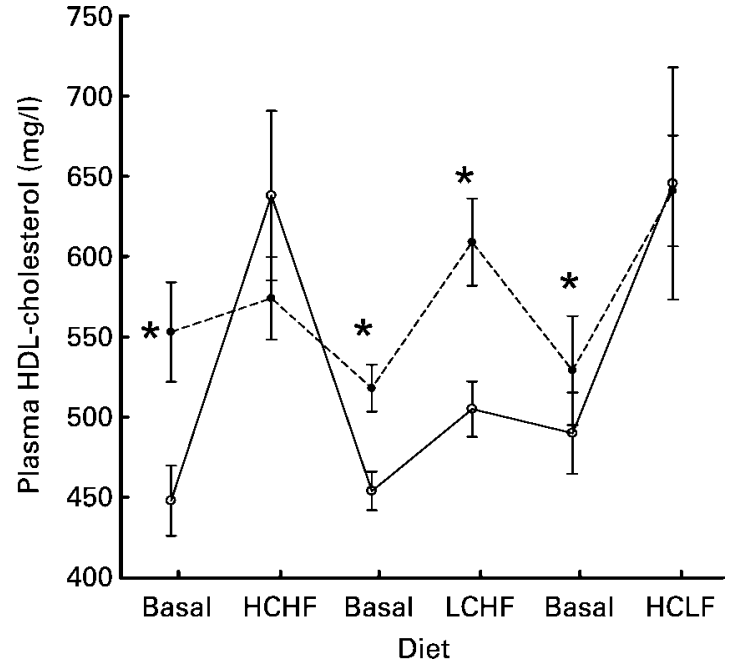

Fig. 2. Plasma HDL-cholesterol concentrations of susceptible $\left(-\mathrm{O}_{-}\right)$and resistant $(--)$lines of laboratory opossums (Monodelphis domestica; ten in each group) in response to diets containing elevated cholesterol and/or fat. The experimental diets were: highcholesterol and high-fat (HCHF); low-cholesterol and high-fat (LCHF); high-cholesterol and low-fat (HCLF) (see Table 1). Mean values are shown, with standard errors of the mean represented by vertical bars. * Mean values for susceptible and resistant lines were significantly different $(P<0.05)$.

animals were more than in resistant animals when fed the $\mathrm{HCHF}$ diet (37\% increase in high-responding animals $v$. $8 \%$ in low-responding animals; $P<0.05)$ and the differences in HDL-cholesterol between the high and low lines observed at baseline were eliminated during the feeding of these diets. However, the differences in HDL-cholesterol between the two lines when they were fed the highfat and low-cholesterol diet were significant (528 (SEM 19.4) $\mathrm{mg} / \mathrm{l}$ in susceptible animals $v$. 630 (SEM 18) $\mathrm{mg} / \mathrm{l}$ in resistant animals) $(P<0 \cdot 01$; Fig. 2$)$.

\section{Effect of dietary cholesterol and/or fat on plasma triacylglycerols}

Plasma triacylglycerol concentrations did not differ between animals of the susceptible and resistant lines when fed the basal diet (200 (SEM 10) mg/l in the susceptible line v. 220 (SEM 10) $\mathrm{mg} / \mathrm{l}$ in the resistant line). The values for plasma triacylglycerols varied considerably after the experimental diets. Plasma triacylglycerols in animals of the susceptible line fed the HCHF and low-cholesterol and high-fat diets were 350 (SEM 40) and 276 (SEM 30) $\mathrm{mg} / \mathrm{l}$, respectively. Similarly, plasma triacylglycerols in animals of the resistant line fed the HCHF and lowcholesterol and high-fat diets were 214 (SEM 20) and 300 (SEM 20) mg/l, respectively. However, there were no significant differences between the susceptible and resistant lines when feeding the experimental diets.

\section{Effect of sex on the diet-induced hypercholesterolaemia in susceptible and resistant lines of opossums}

Table 3 presents plasma and lipoprotein lipid levels for male and female opossums when feeding the basal diet and the HCHF diet. We used seven males and seventeen females from the susceptible line and ten males and sixteen females from the resistant line on the basis of availability. Plasma and lipoprotein cholesterol and plasma triacylglycerol concentrations did not differ between male and female opossums within the same line when fed either the basal or the HCHF diet. Plasma and VLDL+LDLcholesterol concentrations of both male and female opossums of the susceptible line were significantly higher $(P<0.001)$ than plasma and VLDL+LDL-cholesterol concentrations of both male and female opossums of the resistant line. Males in the susceptible line tended to have higher plasma and lipoprotein cholesterol concentrations than females. The difference in HDL-cholesterol concentration between males and females in the susceptible line group was significant $(P=0 \cdot 05)$.

\section{Effect of type of dietary fat with reduced levels of cholesterol on diet-induced hypercholesterolaemia in susceptible opossums}

Table 4 presents the data for effect of type of dietary fat on plasma cholesterol concentrations in opossums of the susceptible line. The animals consumed these diets for 4

Table 3. Effect of sex on diet-induced hypercholesterolaemia in high- and low-responding opossums (Monodelphis domestica) $\dagger$ (Mean values and standard errors of the mean)

\begin{tabular}{|c|c|c|c|c|c|c|c|c|c|c|}
\hline \multirow[b]{2}{*}{ Diet } & \multirow[b]{2}{*}{ Group } & \multirow[b]{2}{*}{ Sex } & \multicolumn{2}{|c|}{$\begin{array}{l}\text { Plasma choles- } \\
\text { terol }(\mathrm{mg} / \mathrm{l})\end{array}$} & \multicolumn{2}{|c|}{$\begin{array}{l}\text { Plasma triacyl- } \\
\text { glycerols (mg/l) }\end{array}$} & \multicolumn{2}{|c|}{$\begin{array}{l}\text { HDL-choles- } \\
\text { terol (mg/l) }\end{array}$} & \multicolumn{2}{|c|}{$\begin{array}{c}\text { VLDL+LDL- } \\
\text { cholesterol (mg/l) }\end{array}$} \\
\hline & & & Mean & SEM & Mean & SEM & Mean & SEM & Mean & SEM \\
\hline \multirow[t]{4}{*}{ Basal } & \multirow[t]{2}{*}{ High } & Male $(n 17)$ & 660 & 24 & 206 & 07 & 449 & 14 & 211 & 13 \\
\hline & & Female $(n 7)$ & 679 & 61 & 203 & 22 & 497 & 40 & 181 & 22 \\
\hline & \multirow[t]{2}{*}{ Low } & Male $(n 10)$ & 681 & 23 & 230 & 11 & 500 & 14 & 181 & 16 \\
\hline & & Female ( $n 16)$ & 721 & 35 & 189 & 09 & 549 & 30 & 171 & 12 \\
\hline \multirow[t]{4}{*}{$\mathrm{HCHF}$} & \multirow[t]{2}{*}{ High } & Male $(n 17)$ & $7688^{*}$ & 947 & 351 & 33 & $677^{*}$ & 38 & $7011^{*}$ & 967 \\
\hline & & Female $(n 7)$ & $5491^{*}$ & 1060 & 294 & 49 & 526 & 69 & $4966^{\star}$ & 1022 \\
\hline & \multirow[t]{2}{*}{ Low } & Male $(n 10)$ & 817 & 43 & 248 & 11 & 557 & 26 & 260 & 26 \\
\hline & & Female $(n 16)$ & 861 & 36 & 195 & 14 & 595 & 18 & 273 & 23 \\
\hline
\end{tabular}

$\mathrm{HCHF}$, high-cholesterol and high-fat.

* Mean values were significantly higher $(P<0.001)$ than those for males and females in the low responding group fed the HCHF diet. Mean values for $\mathrm{HDL}$-cholesterol in males were significantly higher than females in the high-responding group $(P=0.05)$.

†For details of diets and procedures, see Table 1 and p. 64. 
Table 4. Effect of type of dietary fat with reduced levels of cholesterol on diet-induced hypercholesterolaemia in highresponding opossums (Monodelphis domestica) $†$

(Mean values and standard errors of the mean)

\begin{tabular}{|c|c|c|c|c|c|c|}
\hline \multirow[b]{2}{*}{ Diet } & \multicolumn{2}{|c|}{$\begin{array}{l}\text { Plasma cholesterol } \\
(\mathrm{mg} / \mathrm{l})\end{array}$} & \multicolumn{2}{|c|}{$\begin{array}{l}\text { HDL-cholesterol } \\
(\mathrm{mg} / \mathrm{l})\end{array}$} & \multicolumn{2}{|c|}{$\begin{array}{c}\text { VLDL+LDL- } \\
\text { cholesterol (mg/l) }\end{array}$} \\
\hline & Mean & SEM & Mean & SEM & Mean & SEM \\
\hline Basal $(n 50)$ & 654 & 14 & 548 & 12 & 107 & 06 \\
\hline Low-cholesterol and high-lard $(n 10)$ & 970 & 61 & 530 & 31 & 440 & 36 \\
\hline Low-cholesterol and high-coconut-oil ( $n$ 10) & 726 & 35 & 528 & 34 & 198 & 39 \\
\hline High-cholesterol and high-coconut-oil $(n 10)$ & $6347^{*}$ & 583 & $843^{*}$ & 120 & $5503^{*}$ & 703 \\
\hline High-cholesterol and high-lard (HCHF) ( $n$ 10) & $7004^{*}$ & 1120 & $885^{\star}$ & 83 & $6110^{*}$ & 1143 \\
\hline
\end{tabular}

$\mathrm{HCHF}$, high-cholesterol and high-fat.

* Mean values were significantly different from cholesterol values for other diets $(P<0.01)$

†For details of diets and procedures, see Table 1 and p. 64

weeks. The type and amount of saturated fatty acids (lard or coconut oil) had similar effects on the plasma and lipoprotein cholesterol concentrations in susceptible opossums. When dietary cholesterol was fed at $7 \cdot 1 \mathrm{~g} / \mathrm{kg}$ with either the coconut oil or lard, the animals responded with extreme increases in plasma and VLDL+LDL-cholesterol concentrations $(P<0.05)$. However, feeding a lower concentration of cholesterol $(3.55 \mathrm{~g} / \mathrm{kg})$ with coconut oil or lard eliminated the response in plasma and lipoprotein cholesterol completely.

\section{Discussion}

There is considerable variability in the plasma cholesterol response to dietary cholesterol and fat among species and among individuals within the same species (Kushwaha \& McGill, 1997). The high-responding individuals within the same species have a greater increase in plasma cholesterol levels than low-responding individuals. The metabolic mechanisms responsible for the differences in plasma cholesterol responses to dietary lipids differ among various species of animals (McGill \& Kushwaha, 1995). The differences in dietary responses are generally due to dietary cholesterol, dietary fat, or a combination of both. As a consequence of the results reported in the present paper, we are using the laboratory opossum model to identify genetic and metabolic markers for individual variability in plasma cholesterol response to dietary lipids. The uniqueness of this model is that it has extreme genetically controlled variability in responsiveness to dietary lipids, and a great proportion of the genetic variability in responsiveness is due to a single major gene (Rainwater et al. 2001). The present studies were conducted to determine whether the major gene for dietary response detected by genetic analysis in opossums is responsive to dietary cholesterol, dietary fat, or a combination of both. The results demonstrated that the major gene is responsive to dietary cholesterol but not to fat. However, it responds more consistently and more extremely to dietary cholesterol when a high level of fat is present in the diet.

Cholesterol feeding in human subjects also causes an increase in VLDL+LDL-cholesterol, and this increase in LDL is much higher in some individuals than in others (Beynen et al. 1989). Selective breeding in baboons has also produced high and low lines that differ in plasma cholesterol response to dietary cholesterol and fat (McGill et al. 1988). Baboons from a high-responding line increased their VLDL+LDL-cholesterol much more than baboons from a low-responding line when challenged with a HCHF diet (McGill et al. 1988). Dietary cholesterol increased plasma HDL-cholesterol to a small extent, but fat with saturated fatty acids consistently increased HDLcholesterol in these lines of baboons (McGill et al. 1988). Similarly, in laboratory opossums the dietary cholesterol with or without saturated fat increased HDLcholesterol levels, but the increase in VLDL+LDL-cholesterol was considerably more than the increase in HDLcholesterol. Dietary fat selectively increased HDL-cholesterol in both high- and low-responding opossums; however, the increase in HDL-cholesterol in low-responding opossums was higher than in high-responding opossums. Our studies of baboons have also suggested that dietary cholesterol and dietary saturated fat have different effects on metabolic processes (Kushwaha et al. 1991, 1994). In addition, Mott et al. (1992) have suggested that cholesterol and saturated fat have different effects on the hepatic cholesterol and bile acid metabolism in baboons. According to their results, dietary cholesterol elevated hepatic cholesterol content and bile acid secretion, whereas saturated fat decreased hepatic cholesterol content and bile acid secretion. These studies have led to the conclusion that the genes that regulate the cholesterolaemic response to dietary cholesterol are different from those that regulate response to dietary fat in baboons. We have not conducted metabolic studies in opossums, but since their plasma lipoprotein cholesterol responses to dietary fat and dietary cholesterol are similar to those in baboons, it is probable that plasma cholesterol responses to dietary cholesterol and dietary fat are regulated by different genes.

In previous studies we examined metabolic differences between susceptible and resistant opossums (Kushwaha et al. 2001). These studies suggested that susceptible and resistant strains differ in hepatic acyl-coenzyme A:cholesterol acyltransferase (ACAT) and sterol 27-hydroxylase activity. The activity of ACAT was higher in the susceptible line, whereas the activity of sterol 27-hydroxylase was higher in the resistant strain and was induced in response to dietary lipids. Differences in ACAT activity may affect cholesterol absorption and hepatic lipoprotein secretion (Kushwaha \& McGill, 1997). Similarly, 
differences in sterol 27-hydroxylase may affect bile acid synthesis and cholesterol metabolism in the liver and extra-hepatic tissues and may modulate diet-induced hyperlipidaemia. Further studies are needed to determine whether ACAT or sterol 27-hydroxylase induces these differences in response to diet between susceptible and resistant opossums.

In the present study, the addition of fat to the diet exacerbated the plasma cholesterol response to dietary cholesterol in high-responding but not in low-responding opossums. However, fat alone did not have any effect on VLDL+ LDL-cholesterol concentrations in either stock of opossums. Two types of saturated fats (lard and coconut oils) had similar effects on plasma cholesterol response in high responders. The reduction in dietary cholesterol to onehalf that of the HCHF diet blunted the response to diets containing lard or coconut oil. These results are consistent with those from other animals and human subjects. In hamsters and other animal species, when dietary fat is added without added cholesterol, there is little effect on plasma and VLDL+LDL-cholesterol. However, in the presence of dietary cholesterol and fat, plasma and VLDL+LDLcholesterol concentrations are elevated (Spady \& Dietschy, 1988; Dietschy et al. 1993). In hamsters, excess dietary cholesterol also increases HDL-cholesterol (McConihay et al. 2001; Souidi et al. 2001). These studies are also consistent with results from human studies, in which the effect of dietary cholesterol on plasma cholesterol concentrations was not modified by the nature and amount of dietary fat in normolipidaemic human subjects (Kestin et al. 1989). In other human studies, the lipaemic effect of saturated fat was variable. Clifton et al. (1990) demonstrated that dietsensitive human subjects had a greater response to dietary cholesterol, dietary fat or both as compared with modestly hypercholesterolaemic subjects. Fielding et al. (1995) observed considerable variability in plasma cholesterol response to dietary fat and cholesterol, but an increase in saturated fat in place of polyunsaturated fat had no or little effect on the cholesterolaemic effect of diet in human subjects. The presence of a mixed population of high, moderate and low human responders may be a major contributor to the variable results.

In summary, the present results demonstrate that the major gene detected by previous genetic analysis in laboratory opossums is responsive to dietary cholesterol and not to fat. However, dietary fat amplifies the effect of dietary cholesterol, especially in high-responding opossums. Dietary fat increases plasma HDL-cholesterol in both high- and low-responding opossums. The variable effects of dietary cholesterol and dietary fat in laboratory opossums are consistent with the hypothesis that genetic mechanisms that modulate the effect of dietary cholesterol on plasma lipoprotein cholesterol concentrations are different from those that modulate the effect of dietary fat. The many-fold difference in non-HDL-cholesterol concentrations between high- and low-responding opossums, and the existence of a single major gene responsible for these genetic differences in non-HDL-cholesterol concentrations whilst being fed the high-cholesterol diet establishes the laboratory opossum as a unique model for investigating the metabolic and genetic basis for dietary cholesterol responsiveness.

\section{Acknowledgements}

The present study was supported by a grant (no. R01 RR15009) from the National Center for Research Resources, National Institutes of Health, and a grant from the Robert J. Kleberg Jr and Helen C. Kleberg Foundation.

\section{References}

Beynen AC, Katan MB \& Van Zutphen LFM (1989) Hypo- and hyperresponders: individual differences in the response of serum cholesterol concentration to changes in diet. Adv Lipid Res 22, 115-171.

Clifton PM, Kestin M, Abbey M, Drysdale M \& Nestel PJ (1990) Relationship between sensitivity to dietary fat and dietary cholesterol. Arteriosclerosis 10, 394-401.

Dietschy JM, Turley SD \& Spady DK (1993) Role of liver in the maintenance of cholesterol and low density lipoprotein homeostasis in different species, including humans. J Lipid Res 34, $1637-1659$.

Fielding CJ, Havel RJ, Todd KM, Yeo KE, Schloetter MC, Weinberg V \& Frost PH (1995) Effects of dietary cholesterol and fat saturation on plasma lipoproteins in an ethnically diverse population of healthy young men. J Clin Invest 95, 611-618.

Kestin M, Clifton PM, Rouse IL \& Nestel PJ (1989) Effect of dietary cholesterol in normolipidemic subjects is not modified by nature and amount of dietary fat. Am J Clin Nutr 50, 528-532.

Kushwaha RS \& McGill HC Jr (1997) Mechanisms controlling lipemic responses to dietary lipids. World Rev Nutr Diet 80, $82-125$.

Kushwaha RS, McMahan CA, Mott GE, Carey KD, Reardon CA, Getz GS \& McGill HC Jr (1991) Influence of dietary lipids on hepatic mRNA levels of proteins regulating plasma lipoproteins in baboons with high and low levels of large high density lipoproteins. J Lipid Res 32, 1929-1940.

Kushwaha RS, Reardon CA, Getz GS, Lewis DS, Rice KS, Carey KD \& McGill HC Jr (1994) Metabolic mechanisms for responses to dietary cholesterol and fat in high and low LDL responding baboons (Papio sp.). J Lipid Res 35, 633-643.

Kushwaha RS, VandeBerg JF, Jackson EM \& VandeBerg JL (2001) High and low responding strains of laboratory opossums differ in sterol 27-hydroxylase and acyl-coenzyme A:cholesterol acyltransferase activities on a high cholesterol diet. $J$ Nutr Biochem 12, 664-673.

Lipid Research Clinics Program (1974) Manual of Laboratory Operations, vol. 1, Lipid and Lipoprotein Analysis. DHEW Publication no. (NIH) 75-628, p. 56. Washington, DC: US Government Printing Office.

McConihay JA, Horn PS \& Woollett LA (2001) Effect of maternal hypercholesterolemia on fetal sterol metabolism in the Golden Syrian hamster. J Lipid Res 42, 1111-1119.

McGill HC Jr \& Kushwaha RS (1995) Individuality of lipemic responses to diet. Can J Cardiol 11, Suppl. G, 15G-27G.

McGill HC Jr, McMahan CA, Mott GE, Marinez YN \& Kuehl TJ (1988) Effects of selective breeding on the cholesterolemic responses to dietary saturated fat and cholesterol in baboons. Arteriosclerosis 8, 33-39.

Manis GS, Hubbard GB, Hainsey BM, Ely JJ, VandeBerg JL \& Stone WH (1992) Effects of chronic blood loss in a marsupial (Monodelphis domestica). Lab Anim Sci 42, 567-571.

Mott GE, Jackson EM \& McMahan CA (1992) Effects of dietary cholesterol, type of fat, and sex on bile lipid composition of adult baboons. Am J Clin Nutr 56, 511-516. 
Rainwater DL \& VandeBerg JL (1992) Dramatic differences in lipoprotein composition among gray short-tailed opossums (Monodelphis domestica) fed a high cholesterol/saturated fat diet. Biochim Biophys Acta 1126, 159-166.

Rainwater DL, Kammerer CM, Singh ATK, Moore PH, Poushesh M, Shelledy WR, VandeBerg JF, Robinson ES \& VandeBerg JL (2001) Genetic control of lipoprotein phenotypes in the laboratory opossum, Monodelphis domestica. GeneScreen 1, 117-124.

Robinson ES \& VandeBerg JL (1994) Blood collection and surgical procedures for the laboratory opossum (Monodelphis domestica). Lab Anim Sci 44, 63-68.

Souidi M, Combettes-Souverain M, Milliat F, Eckhardt ER,
Audas O, Dubrac S, Parquet M, Ferezou J \& Lutton C (2001) Hamsters predisposed to sucrose-induced cholesterol gallstones (LPN strain) are more resistant to excess dietary cholesterol than hamsters that are not sensitive to cholelithiasis induction. J Nutr 131, 1803-1811.

Spady DK \& Dietschy JM (1988) Interaction of dietary cholesterol and triglycerides in the regulation of hepatic low density lipoprotein transport in the hamster. J Clin Invest 81, 300-309. VandeBerg JL (1999) The laboratory opossum, Monodelphis domestica. In UFAW Handbook on the Care and Management of Laboratory Animals, 7th ed., vol. 1. Terrestrial Vertebrates, pp. 193-209 [T Poole and P English, editors]. Oxford, UK: Blackwell Science Ltd. 